\title{
Specifying the waveforms for the calibration of CISPR 16-1-1 measuring receivers
}

\author{
Marco A. Azpúrua, Senior Member, IEEE, Marc Pous, Member, IEEE, and Ferran Silva Fellow, IEEE
}

\begin{abstract}
Ensuring the measuring receiver's compliance with the specifications given in CISPR 16-1-1 is mandatory. This is achieved through traceable calibration of the instrument regarding its standard requirements. Incidentally, the receiver's response to pulses of the weighting detectors is the most challenging aspect that must be calibrated. The calibration method requires generating broadband pulses of a given impulse area and at certain repetition frequencies. Such pulses must deliver a known quasi-peak level and have a flat spectrum over the frequency band under consideration. Whereas the standard states the tolerable uncertainties for the pulse characteristics, it does not stipulate their exact waveform. This void is significant because it hinders the reproducibility of the calibration method, it forbids to completely describe the CISPR 16-1-1 pulse generator and, it truncates the traceability chain. This paper proposes a parametric model for pulsed waveforms that are compliant with the requirements established for the calibration of the receivers' response to pulses. The model is numerically solved and evaluated for producing a waveform vector that can be transformed into a reference voltage signal using arbitrary function generators. The validity of the proposed waveforms is experimentally verified in terms of its time domain characteristics and its frequency spectrum.
\end{abstract}

Index Terms-Calibration, electromagnetic interference, measuring receiver, pulse response, waveform measurements.

\section{INTRODUCTION}

$\mathrm{T}$ $\mathrm{HE}$ measuring receiver is a fundamental element in conducted and radiated electromagnetic emissions testing. Accordingly, the standard definition given in the CISPR 16-11 Ed. 4.0 states it is an "instrument such as a tunable voltmeter, an electromagnetic interference receiver, a spectrum analyzer or a Fast Fourier Transform (FFT) based measuring instrument, with or without preselection, that meets the relevant parts of this standard" [1]. Henceforth, the standard CISPR 16-1-1 does not provide guidelines for any implementation of a measuring receiver but several requirements that the measuring instrument must fulfill under a "black-box" approach [2]. In particular, the baseline (minimum) requirements that should be ensured for any measuring receiver are given in terms of four parameters,

This work was supported in part by the EURAMET 15RPT01 Research Project (the EMPIR is jointly funded by the EMPIR participating countries within EURAMET and the European Union), in part by the Spanish Ministerio de Economía, Industria y Competitividad, under Project TEC2016-79214-C32-R (AEI/FEDER, UE), in part by the Secretaria d'Universitats i Recerca del namely, Voltage Standing Wave Ratio (VSWR), Sine wave voltage accuracy, Response to pulses, and Selectivity [1]-[3].

Therefore, it is mandatory to assess those baseline parameters above to verify if test receivers satisfy the target figures of merit and then, it is possible to certify whether a measuring receiver complies with CISPR 16-1-1. However, considering there might be plenty of different types of implementations of measuring receivers, one could wonder how is it possible to ensure that those requirements are fulfilled for every different brand and model of the test receiver?

As an answer, the CISPR 16-1-1 indicates in its normative annex $\mathrm{K}$ that “....the demonstration of compliance of measuring receivers with specifications defined in this standard can be provided through the manufacturer's calibration process or the procedures and measuring equipment defined in this standard". In that sense, a recent interpretation sheet prepared by the subcommittee CISPR A clarified this means that "it is permissible to use either the manufacturer's calibration process or a calibration laboratory's own process that is applying the procedures and measuring equipment defined in this standard". In any case, the standard transfers the responsibility of using either approach to the user, i.e., the calibration laboratory [4].

In this regard, a laboratory capable of calibrating $\mathrm{RF} /$ microwave test equipment, such as spectrum analyzers, is technically proficient to perform an assessment of most of the baseline parameters of a measuring receiver. This is because the corresponding calibration methods and setups for determining the level accuracy, the frequency response or the selectivity accuracy are well-established. In fact, the only requirement is using general purpose standard reference instruments, such as RF power meters, power sensors, signal generators, vector network analyzers (VNA), step attenuators and splitters [5]-[7].

Nonetheless, for calibrating the response to pulses of the weighting detectors, the established method involves using a baseband (nanosecond) pulse generator capable of delivering a set of pulses with a fixed impulse area and repetition frequency [8]. Such pulses are meant to produce a known quasi-peak voltage level and to have a flat spectrum over the frequency band under consideration (CISPR bands A to D). However, whereas the standard states the tolerable uncertainties for the specified pulse characteristics, it does not stipulate the exact

Departament d'Economia i Coneixement de la Generalitat de Catalunya, and in part by the European Social Fund. (Corresponding author: Marco A. Azpúrua.)

M. A. Azpúrua, M. Pous, and F. Silva are with the Electromagnetic Compatibility Group, Electronic Engineering Department, Universitat Politècnica de Catalunya, Barcelona, Spain (e-mail: marco.azpurua@upc.edu). 
waveform of the pulses. This void in the standard is significant because it hinders the reproducibility of the receiver's calibration method, it does not allow to completely describe the CISPR 16-1-1 pulse generator and, it truncates the traceability chain of the measuring receiver.

Currently, to the best of authors' knowledge, Schwarzbeck is the only manufacturer that provides specific pulse generators as a commercial solution for fulfilling CISPR 16-1-1 calibration requirements. This means the pulse generators IGUU 2916 and IGUU 2918 are both considered, by default, the sole standard for the calibration of the receivers' pulse response. This factual situation could be attributed to the ill-defined waveform characteristics of the reference pulses and it contradicts to some extent the spirit of CISPR 16-1-1 annex K.

To address the limitation above, the first step is to define a complete set of waveform characteristics for the standard reference pulses. Provided such compliant waveforms, calibration laboratories would be able to reproduce easily the standard pulses using general purpose signal and arbitrary function generators. In consequence, that would enable many calibration laboratories to completely cover the baseline magnitudes that must be assessed in CISPR 16-1-1 measuring receivers.

For the reasons above, this paper first studies the characteristics of the CISPR 16-1-1 calibration pulses and proposes a parametric model for describing the waveforms that are compliant with the requirements (Section II). Then, those waveforms are numerically solved and evaluated for producing a reference voltage vector that can be synthesized using arbitrary function generators (Section III). The validity of the proposed waveforms is experimentally verified and compared with the waveforms produced by a commercial EMI Calibration Pulse Generator (Section IV).

\section{COMPliant WAVEForms For the CALIBRATION OF THE MEASURING RECEIVER's PULSE RESPONSE}

The calibration of the absolute and relative pulse response for the peak, quasi-peak (QP), average (AV) and RMS detectors is meant to ensure the measuring receiver is accurately detecting broadband-impulsive noise. This is important because the pass/fail decision regarding emissions testing is made upon the weighting detector levels. Therefore, CISPR 16-1-1 defines as mandatory the receivers' compliance with the response to pulses requirements and this must be attested through periodic calibrations and routinely internal verifications.

In that sense, the calibration of the response to pulses consists of measuring with the receiver the spectrum of the reference pulses and then verifying the detectors levels do not deviate significantly from the CISPR 16-1-1 requirements. The required setup is simple, as shown in Fig 1. Arranging this calibration setup would be trivial if it were not for the convoluted definition of the pulses given in the CISPR 16-1-1 standard.

In what follows, the standard specifications for the reference pulses will be presented and then a parametric model for defining a compliant waveform will be developed.

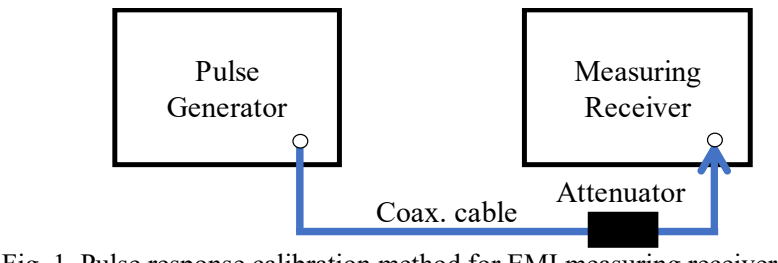

Fig. 1. Pulse response calibration method for EMI measuring receivers.

\section{A. Standard requirements}

In the current version of CISPR 16-1-1 (Ed. 4.0), the requirements given regarding the calibration pulses are limited to their amplitude level, frequency flatness (spectrum uniformity), impulse area, and pulse repetition frequency.

On the one hand, the reference pulses' spectrum must be flat in the frequency band under assessment (CISPR bands A, B, and $\mathrm{C} / \mathrm{D}$ ) with a level equivalent to a tone having an RMS voltage of $2 \mathrm{mV}$, which means $66 \mathrm{~dB}(\mu \mathrm{V})$ at $50 \Omega$ source impedance and in open circuit conditions. In this regard, the spectrum of the pulses is considered sufficiently flat if the deviation of the spectrum amplitude is less than $\pm 2 \mathrm{~dB}$ relative to its value for the lower frequencies within the band. On the other hand, the spectrum above the upper limit of the frequency band shall be limited at least $10 \mathrm{~dB}$ down at twice the upper frequency.

Concerning the impulse area, $A_{\text {imp }}$, CISPR 16-1-1 give the specifications that are reproduced in Table I and indicates that it shall be known within $\pm 0.5 \mathrm{~dB}$ and the repetition frequency, $f_{\text {rep }}$, to within about $1 \%$. These flat pulses are applied for different repetition rates in order to obtain a certain ratio between peak, QP, AV, and RMS detectors, weighting the repetitiveness of the measured interferences [1].

TABLE I. STANDARD IMPULSE AREA SPECIFICATION

\begin{tabular}{ccc}
\hline \hline CISPR frequency band & $\boldsymbol{A}_{\text {imp }}[\mu \mathrm{Vs}]$ & $\boldsymbol{f}_{\text {rep }}{ }^{\mathrm{a}}[\mathrm{Hz}]$ \\
\hline \hline $\mathrm{A}(9 \mathrm{kHz}-150 \mathrm{kHz})$ & 13.5 & $1,2,5,10, \mathbf{2 5}, 60,100$ \\
$\mathrm{~B}(150 \mathrm{kHz}-30 \mathrm{MHz})$ & 0.316 & $1,2,10,20, \mathbf{1 0 0}, 1000$ \\
$\mathrm{C} / \mathrm{D}(30 \mathrm{MHz}-1 \mathrm{GHz})$ & 0.044 & $1,2,10,20, \mathbf{1 0 0}, 1000$ \\
\hline
\end{tabular}

${ }^{\mathrm{a}}$ The reference repetition frequency for relative calibration for each CISPR band is highlighted in bold.

Moreover, the standard recognizes that above $1 \mathrm{GHz}$ it is not feasible to generate pulses with the amplitudes and the rise time that would be required. Consequently, CISPR 16-1-1 indicates that: "Above $1 \mathrm{GHz}$, the required impulse area is defined using a pulse-modulated carrier at the frequency of test...". Previously, alternative methods have been proposed for this purpose [2].

Finally, it could be argued that the current standard definition of the calibration pulses is not completely clear. The forthcoming $5^{\text {th }}$ edition of CISPR 16-1-1 (which is expected to be published in June 2019) might rearrange and reword the statement and the table above. On the other hand, the corresponding harmonized (European) standard EN 55016-1-1 is still based upon the $3^{\text {rd }}$ edition of CISPR 16-1-1. Consequently, several and slightly different definitions of the calibration pulses are coexisting nowadays.

The reasons above stress the need to a better, more specific, and clear definition of the calibration pulses. The work presented in the following sections points to that. 


\section{B. Waveforms of a Calibration-Pulse Generator}

During an intercomparison exercise organized in the frame of the Joint Research Project "Development of RF and Microwave Metrology Capability," (RFMicrowave, 15RPT01), an EMI Calibration Pulse Generator type IGUU 2916 manufactured by Schwarzbeck Mess-Elektronik (S/N 164) and provided by TÜBITAK UME was used as a traveling measurement standard.

Particularly, the focus of this intercomparison exercise was to measure the spectrum of the pulses. Nonetheless, complementary waveform measurements were performed using the setup shown in Fig. 1 but replacing the measuring receiver with a real-time sampling oscilloscope.

Figures 2 to 4 show the IGUU 2916 waveforms measured with the oscilloscope DPO5014B from Tektronix after applying the correction factors for compensating the signal path attenuation (cable and a $20 \mathrm{~dB}$ external attenuator). Equivalent time sampling was used at $400 \mathrm{GSa} / \mathrm{s}$ with a bandwidth of $1 \mathrm{GHz} . A_{\text {imp }}$ was equal to $13.3 \mu \mathrm{Vs}, 0.306 \mu \mathrm{Vs}$, and $0.0446 \mu \mathrm{Vs}$ for bands $\mathrm{A}, \mathrm{B}$ and $\mathrm{C} / \mathrm{D}$ respectively, which is satisfactory in terms of the standard specifications.

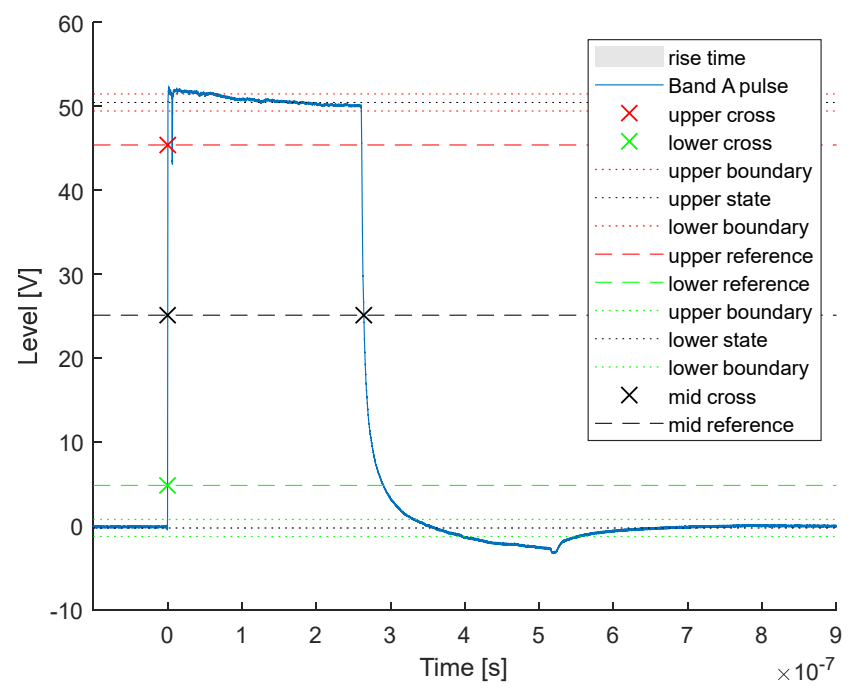

Fig. 2. Calibration pulse generated by the IGUU 2916 for CISPR band A.

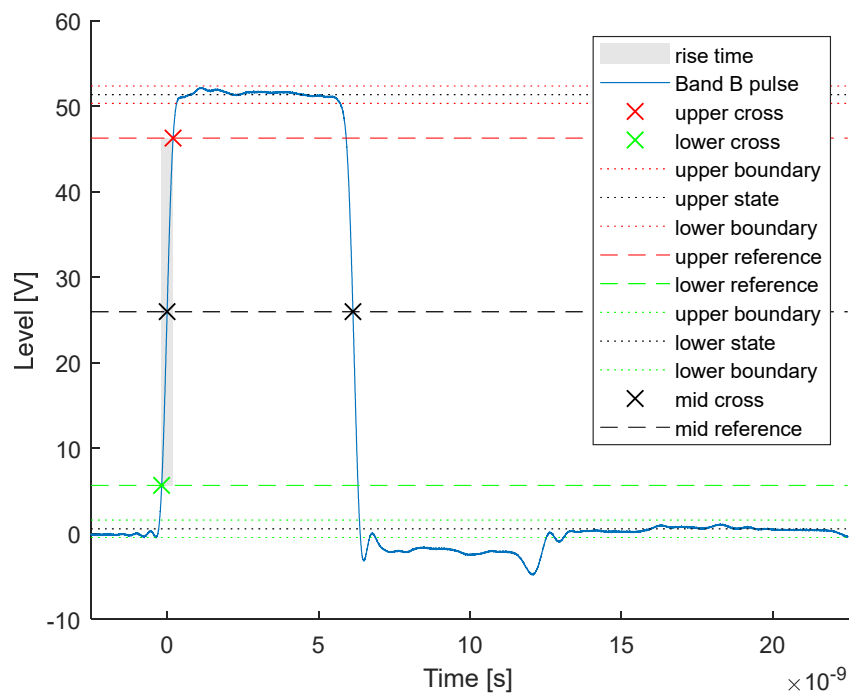

Fig. 3. Calibration pulse generated by the IGUU 2916 for CISPR band B.

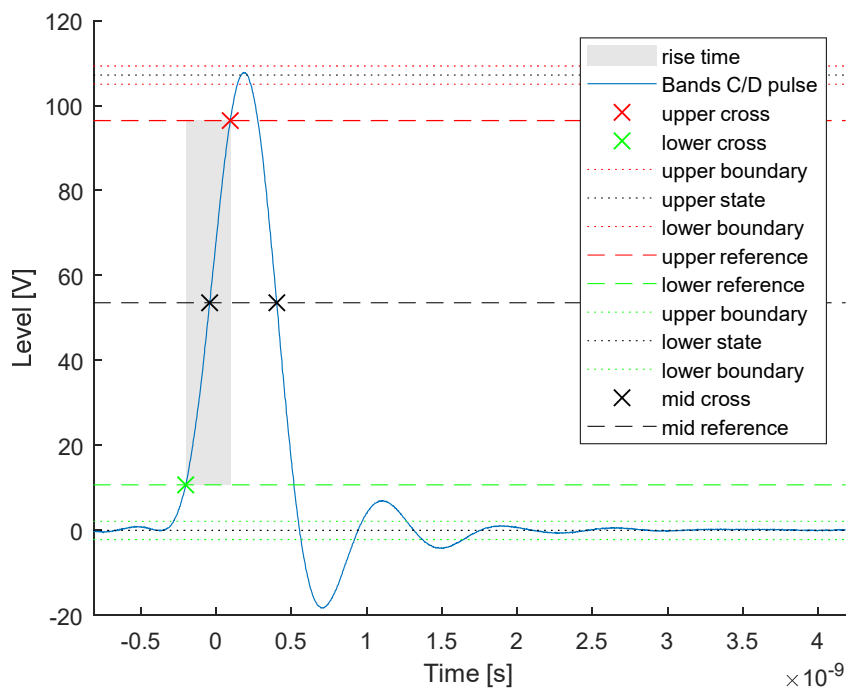

Fig. 4. Calibration pulse generated by the IGUU 2916 for CISPR bands C/D.

TABLE II. PULSE CHARACTERISTICS OF THE IGUU 2916 WAVEFORMS

\begin{tabular}{r|c|c|c}
\hline \multirow{2}{*}{ Characteristic } & \multicolumn{3}{|c}{ CISPR Band } \\
\cline { 2 - 4 } & A & B & C/D \\
\hline \hline Rise time, $t_{r, 10-90 \%}[\mathrm{ps}]$ & 472.40 & 464.81 & 370.32 \\
\hline Fall time, $t_{f, 90-10 \%}[\mathrm{~ns}]$ & 28.67 & 0.39 & 0.24 \\
\hline Pulse width, $\tau[\mathrm{ns}]$ & 263.91 & 6.14 & 0.446 \\
\hline Mean voltage of the upper state, $U_{\text {high }}[\mathrm{V}]$ & 50.47 & 51.36 & 107.17 \\
\hline Max. level, $U_{\max }[\mathrm{V}]$ & 52.14 & 51.93 & 107.20 \\
\hline $0 \mathrm{~dB} /$ decade breakpoint $=1 /(\pi \tau)[\mathrm{MHz}]$ & 1.21 & 51.85 & 713.19 \\
\hline $20 \mathrm{~dB} /$ decade breakpoint $=1 /\left(\pi t_{r}\right)[\mathrm{MHz}]$ & 673.81 & 684.82 & 859.56 \\
\hline Bandwidth, BW=1/t $t_{r}[\mathrm{GHz}]$ & 2.11 & 2.15 & 2.7 \\
\hline \hline
\end{tabular}

Table II includes the pulse characteristics calculated from the beforementioned measurements.

From the results above, it is important to notice:

- In all cases, the measured rise time was relatively similar, in the sub-nanosecond range.

- The rise and fall times were not necessarily equivalent.

- The maximum voltage levels of the calibration pulses are potentially destructive for the inputs of some measuring receivers. In fact, this is warned by the pulse generator manufacturer in its manual.

- The bandwidth of the baseband pulses exceeds the frequency range of the corresponding CISPR bands. For CISPR bands A and B the $0 \mathrm{~dB} /$ decade decay frequency breakpoint is above the maximum frequency of the band. This means the calibration pulses have a significant amount of energy spread in frequencies above the frequency band under assessment.

- If the measured waveforms are considered as approximately rectangular, their impulse area could be estimated as the pulse width times the mean voltage of the upper state, that is, $A_{\text {imp }}=\tau \times U_{\text {high }}$. Accordingly, the difference between the impulse area calculated using the rectangular approximation and the direct integration of the measured waveform area is $0.06 \mathrm{~dB}, 0.17 \mathrm{~dB}$ and $0.66 \mathrm{~dB}$ for CISPR bands $\mathrm{A}, \mathrm{B}$, and $\mathrm{C} / \mathrm{D}$, respectively.

Regarding the measurements above, one could affirm that it would be advisable to have used an oscilloscope of higher 
bandwidth for characterizing such calibration pulses given their short duration and high slew rate. On the other hand, we would like to highlight our goal with this measurement was to identify the fundamental characteristics and drawback of this kind of pulse generator for, then, developing an alternative and more suitable pulse calibration waveform completely based on a parametric model.

\section{Parametric Model}

For defining the suitable waveforms for the measuring receivers' calibration pulses, let's start from the model of a periodic trapezoid pulse, $p(t)$, shown in Figure 5.

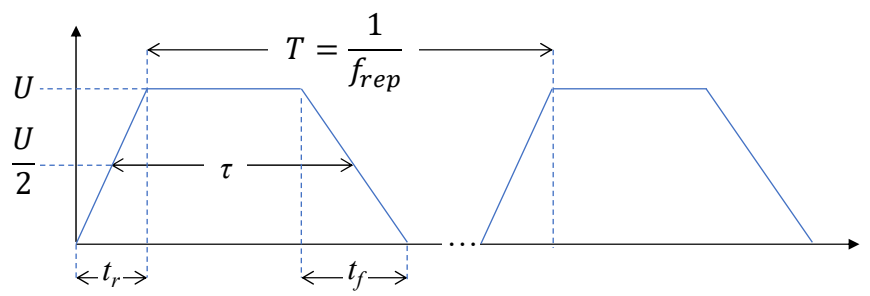

Fig. 5. A generic representation of a trapezoid pulse.

Firstly, the amplitude of the trapezoid can be easily computed from the pulse area requirement, $A_{\text {imp }}$, and the pulse width, $\tau$, as

$$
U=\frac{A_{i m p}}{\tau} \text {. }
$$

However, the pulse width is unknown. In that sense, it is necessary to draw upon the pulse spectrum requirements to extract the remaining pulse timing parameters.

In that sense, it is possible to represent the periodic trapezoid waveform above as a Fourier series, that is,

$$
p(t)=c_{0}+\sum_{n=1}^{\infty}\left|c_{n}\right| \cos \left(n \omega_{r e p} t+\theta_{n}\right)
$$

where $\omega_{\text {rep }}=2 \pi f_{\text {rep }}$. The constant coefficient in (2), $c_{0}$, corresponds to the average value of the waveform over one period of time [9], that is

$$
c_{0}=\frac{1}{T} \int_{0}^{T} p(t) d t=\frac{U \tau}{T}
$$

whereas magnitude and phase of the harmonic components of the Fourier series are given by

$$
c_{n}=\left|c_{n}\right| e^{i \theta_{n}}=\frac{2}{T} \int_{0}^{T} p(t) e^{-i n \omega_{r e p} t} d t
$$

If (4) is solved for the case in which $t_{r}=t_{f}$, then the expressions for the amplitude and the phase of $c_{n}$ are (5) and (6).

$$
\begin{gathered}
\left|c_{n}\right|=2 U \frac{\tau}{T}\left(\frac{\sin \left(\pi n f_{\text {rep }} t_{r}\right)}{\pi n f_{\text {rep }} t_{r}}\right)\left(\frac{\sin \left(\pi n f_{\text {rep }} \tau\right)}{\pi n f_{\text {rep }} \tau}\right) \\
\theta_{n}=-\pi n\left(\tau+t_{r}\right) f_{\text {rep }}
\end{gathered}
$$

Then, for each CISPR band there are a pair of coefficients $c_{l}$ and $c_{u}$ corresponding to the lower and upper frequency components of that band, $f_{l}$ and $f_{u}$, respectively, were $l=f_{l} / f_{\text {rep }}$ and $u=f_{u} / f_{\text {rep }}$.

At this point, the ratio of the amplitude of the coefficients in the extremes of each CISPR frequency band can be used as a measure of the pulse's spectrum flatness, $F_{l, u}$,

$$
F_{l, u}\left(t_{r}, \tau\right)=\frac{\left|c_{l}\right|}{\left|c_{u}\right|}=\frac{u^{2}}{l^{2}}\left|\frac{\sin \left(\pi l f_{r e p} t_{r}\right) \sin \left(\pi l f_{r e p} \tau\right)}{\sin \left(\pi u f_{r e p} t_{r}\right) \sin \left(\pi u f_{r e p} \tau\right)}\right|
$$

which means the flatness is a function of the pulse rise time and the pulse width.

The spectrum of the pulse cannot be perfectly flat, a tolerable deviation of the flatness, $\Delta_{F}$, must be allowed, which means,

$$
20 \log \left[F_{l, u}\left(t_{r}, \tau\right)\right] \leq \Delta_{F}
$$

Therefore, the solution space of (1) and (8) contains the parameters that would comply with the requirements for the calibration pulses. Henceforward, suitable values within the solution space must be numerically calculated.

Still, an infinite number of harmonics are required by (2) to faithfully reproduce the trapezoid waveform of $p(t)$. Certainly, this is against the band limited behavior of the calibration pulse required by the standard.

In view hereof, for limiting the bandwidth of the calibration pulses, the amplitude of their spectrum must decrease with frequency above the upper limit of the frequency band. A certain decay $(10 \mathrm{~dB})$ in the pulse spectrum amplitude is expected at $2 f_{u}$ for ensuring consistent severity in terms of the influence of the receiver intermodulation products. Similarly, the dc and the frequency components above $2 f_{u}$ can be discarded since their energy contribution to the waveform is negligible.

Consequently, the expression of the pulse calibration waveform, $p_{c a l}(t)$, is given by,

$$
p_{\text {cal }}(t)=\sum_{n=1}^{2 u} d_{n}\left|c_{n}\right| \cos \left(n \omega_{\text {rep }} t+\theta_{n}\right)
$$

where $d_{n}$ in the weighting factor for adjusting the amplitude of the $n$-th harmonic components, and it is defined by,

$$
d_{n}=\left\{\begin{array}{cl}
1 & \text { if } 1 \leq n \leq u \\
\frac{D\left(n f_{r e f}\right)}{\left|c_{n}\right|} & \text { if }|n|>u
\end{array}\right.
$$

where $D(f)$ is the decay function that describes the transition between the passband and the stopband regions of a zero-phase low pass filter of unitary gain. In this regard, the above mentioned transition must be continuous, that is, $D\left(f_{u}\right)=\left|c_{u}\right|$. Additionally, it is known that at $f=2 f_{u}$ the amplitude of the pulse spectrum must be attenuated by a factor $\alpha$ with respect to the level at the lower limit of the frequency band, which means, $D\left(2 f_{u}\right)=\alpha\left|c_{u}\right|$. Finally, even if $D(f)$ is not specified, a linear relationship can be assumed for the sake of simplicity. Then, $D(f)$ is given by,

$$
D(f)=\left|c_{u}\right|+\left(\alpha\left|c_{l}\right|-\left|c_{u}\right|\right)\left(\frac{f}{f_{u}}-1\right)
$$


TABle III. SPECIFIEd CALIBRATION PUlSe PARAMETERS

\begin{tabular}{r|c|c|c}
\hline \hline \multirow{2}{*}{ Parameter } & \multicolumn{3}{|c}{ CISPR Band } \\
\cline { 2 - 4 } Lower harmonic index, $l$ & 360 & 1500 & $3 \times 10^{5}$ \\
\hline Upper harmonic index, $u$ & 6000 & $3 \times 10^{5}$ & $10^{7}$ \\
\hline Pulse repetition frequency, $f_{\text {rep }}$ & $25 \mathrm{~Hz}$ & $100 \mathrm{~Hz}$ & $100 \mathrm{~Hz}$ \\
\hline Tolerable deviation of the flatness, $\Delta_{F}$ & $2 \mathrm{~dB}$ & $2 \mathrm{~dB}$ & $2 \mathrm{~dB}$ \\
\hline Margin for the pulse flatness, $M_{F}$ & $0.5 \mathrm{~dB}$ & $0.5 \mathrm{~dB}$ & $0.5 \mathrm{~dB}$ \\
\hline \hline
\end{tabular}

\section{NUMERICAL EVALUATION}

The first step for obtaining a valid solution for the parameters of the calibration pulse is to set a target value for its spectrum flatness. Under the less stringent flatness condition, the compliance with the requirement in (8) would be hardly ensured. In practice, the uncertainty in the pulse generator would decrease the actual pulse's spectrum flatness. Accordingly, a margin for the spectrum flatness, $M_{F}$, must be accounted, and then the objective function is defined as,

$$
\Omega\left(t_{r}, \tau\right)=20 \log \left[F_{l, u}\left(t_{r}, \tau\right)\right]-\Delta_{F}+M_{F}
$$

Then, a suitable approximate solution for the calibration pulse timing parameters $\left(t_{r}^{\prime}, \tau^{\prime}\right)$ is found following a minimization approach of the problem below,

$$
\left|\Omega\left(t_{r}^{\prime}, \tau^{\prime}\right)\right|<\delta \wedge t_{r}^{\prime}>0 \wedge \tau^{\prime}>0
$$

where an allowable absolute error, $\delta$, of less than $10^{-6}$ is defined as acceptable. The numerical resolution of the surface response was $1 \mathrm{ps}$ in each dimension and it was evaluated using the settings in Table III for each CISPR band.

The results are shown in Figures 6 to 8 . We realized that the suitable numerical solutions satisfied the $\rho^{2}=\left(t^{\prime}\right)^{2}+\left(\tau^{\prime}\right)^{2}$ condition. Therefore, fixing a reasonable value for the rise time and solving a suitable pulse width allows for calculating other appropriate combinations of pulse timing parameters. In that sense, the above mentioned figures also display a white rhombus that represents the selected solution for each band.

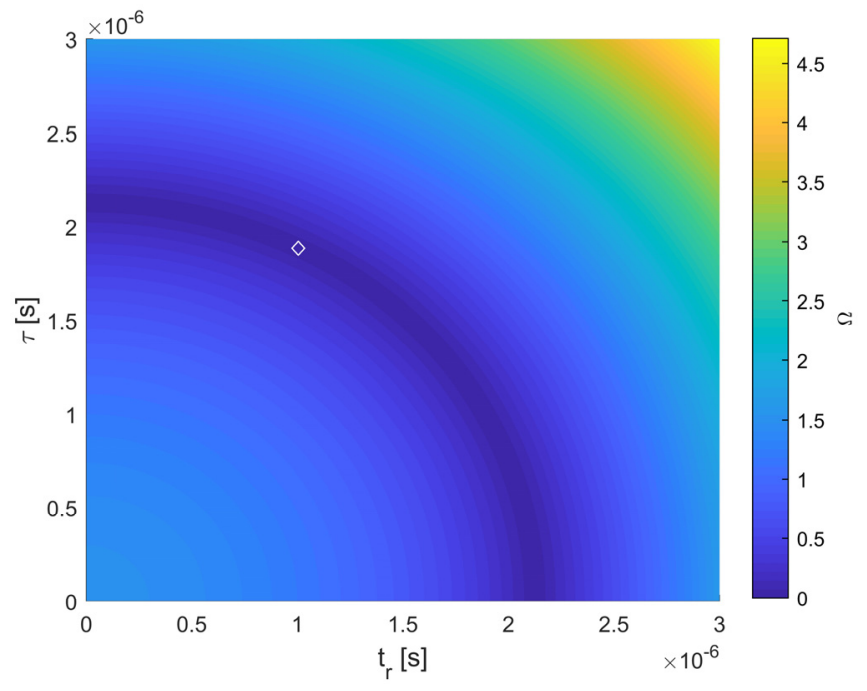

Fig. 6. Surface response of the objective function for CISPR band A.

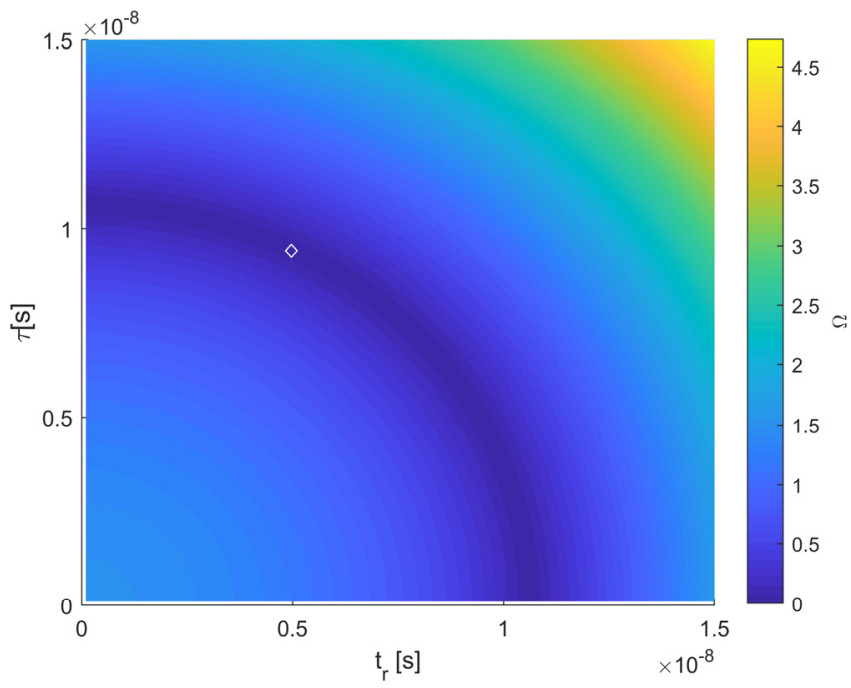

Fig. 7. Surface response of the objective function for CISPR band B.

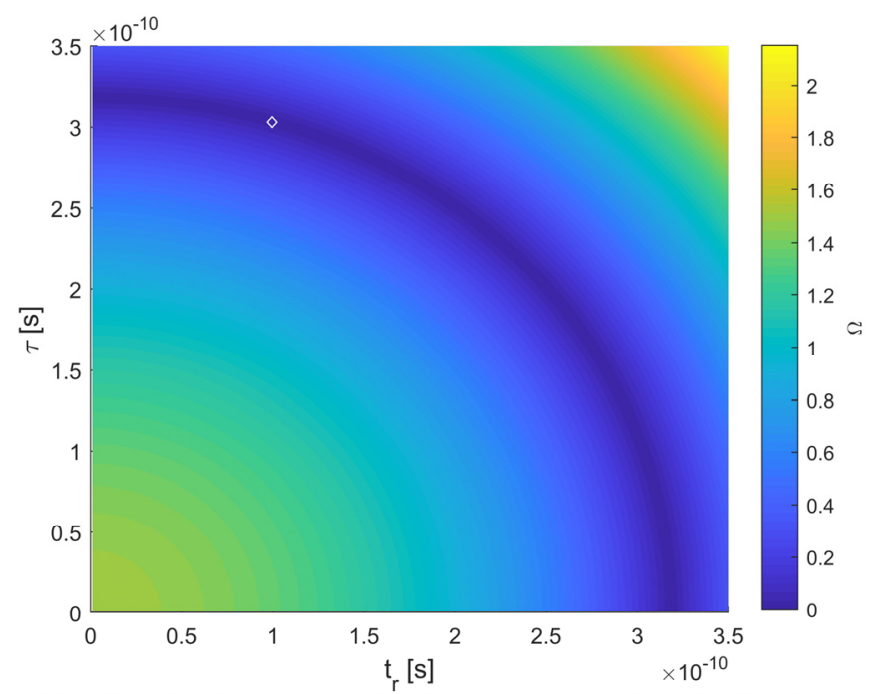

Fig. 8. Surface response of the objective function for CISPR band C/D.

Table IV shows the numerically solved parameters for the calibration pulses in CISPR bands A, B, and C/D. With those parameters, it is possible to evaluate (9) the Fourier series coefficients, $c_{n}$, and the corresponding weighting factors, $d_{n}$, for a factor $\alpha \approx 0.316$, which is equivalent to a $-10 \mathrm{~dB}$ decay. The results of the numerical computation of the calibration pulse waveform are shown in Figures 9 to 11.

TABLE IV. SOLUTION FOR THE CALIBRATION PULSE PARAMETERS

\begin{tabular}{r|c|c|c}
\hline \multirow{2}{*}{ Parameter } & \multicolumn{3}{|c}{ CISPR Band } \\
\cline { 2 - 4 } & A & B & C/D \\
\hline Amplitude of the trapezoid, $U$ & 7.14 & 33.50 & 146.67 \\
\hline Rise time of the trapezoid pulse, $t_{r}$ & $1 \mu \mathrm{s}$ & $5 \mathrm{~ns}$ & $0.1 \mathrm{~ns}$ \\
\hline Pulse width, $\tau$ & $1.89 \mu \mathrm{s}$ & $9.43 \mathrm{~ns}$ & $0.3 \mathrm{~ns}$ \\
\hline \hline
\end{tabular}

Alongside the calibration pulse waveforms, their quasi-peak spectrum is displayed for verifying its compliance with the flatness and band limiting requirements. The quasi-peak amplitude spectrum of the pulses has been calculated following the same processing approach as in compliant time-domain EMI measurements. Consequently, the estimated spectra of the calibration pulses are compliant according to the standard bandwidth selectivity requirements and the weighting detector response defined by the CISPR 16-1-1 [2], [3], [10], [11]. 

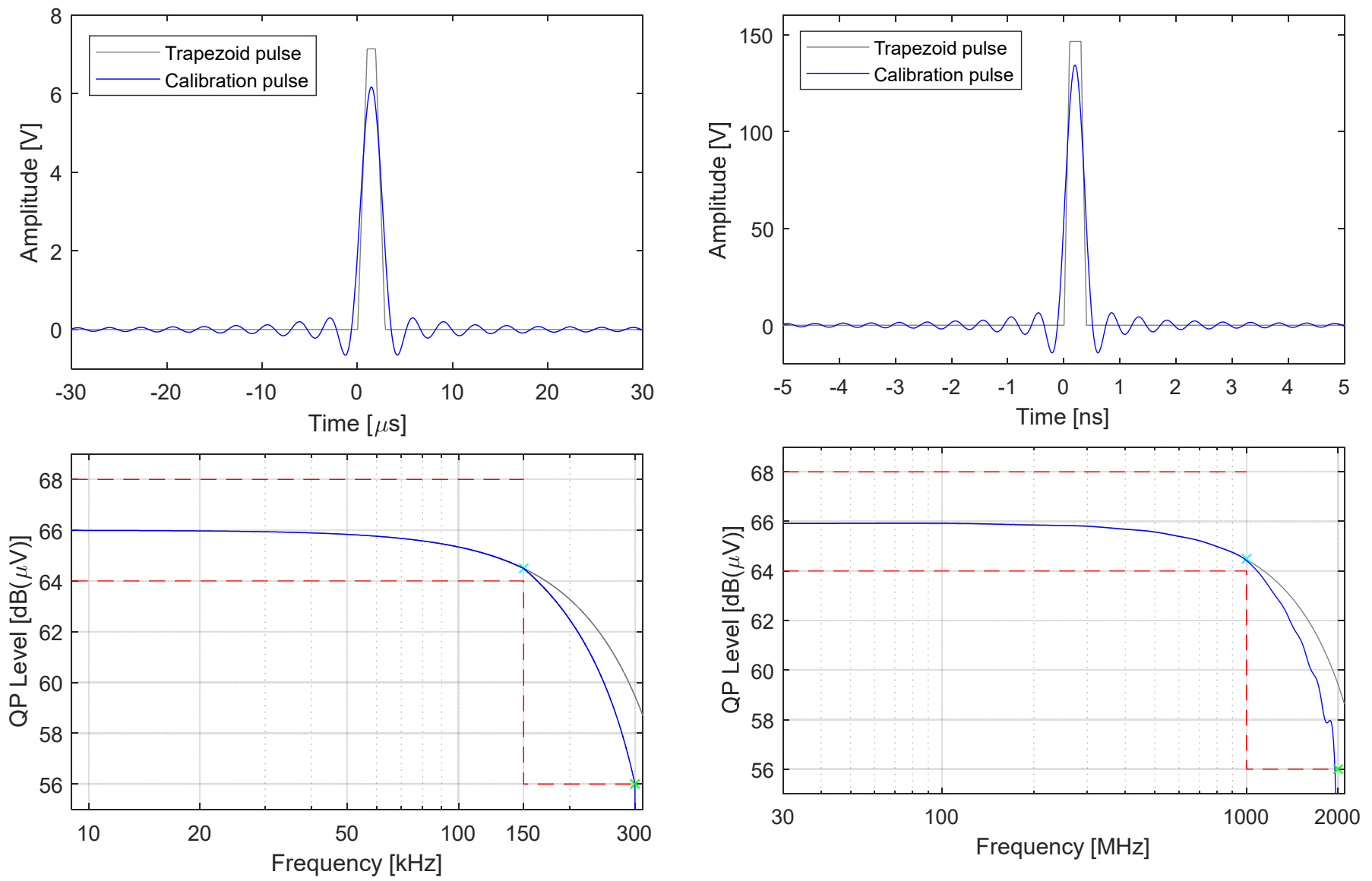

Fig. 9. Calibration pulse for CISPR band A and its frequency spectrum.

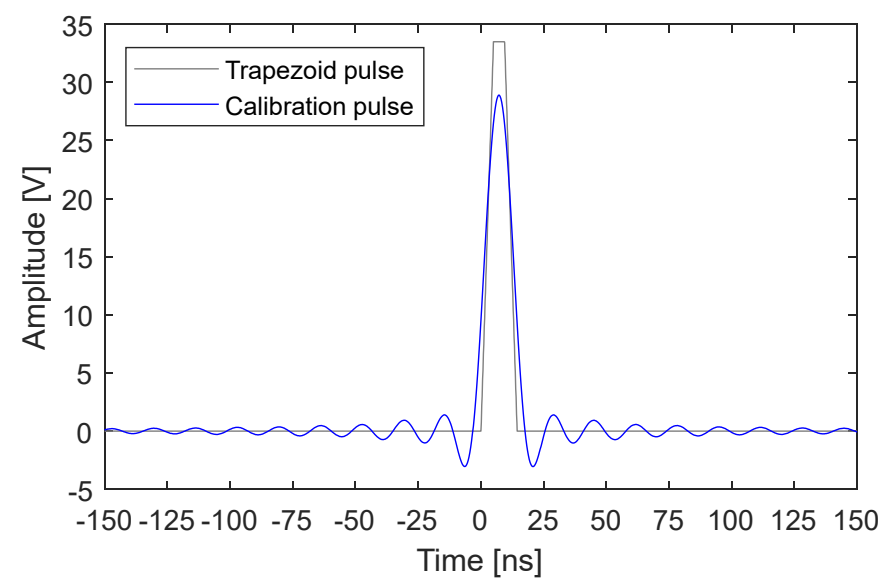

Fig. 11. Calibration pulse for CISPR bands $C / D$ and its frequency spectrum.

Another important aspect to consider regarding the proposed calibration pulses is that, given their spectrum is band limited, their waveforms are spread during the whole pulse period. Nonetheless, most of the pulse energy is concentrated around its peak point, $\left(t_{\text {peak }}, U_{\text {peak }}\right)$. Therefore, in practice, it is convenient to define a criterion for truncating the numeric evaluation of the pulse waveform and, then, defining the pulse duration according to that criterion. The before mentioned truncation of the waveform is responsible for the slight distortion of the pulse spectrum near the upper limit of its frequency components.

Consequently, the Parseval's theorem states the power of a signal calculated in the time domain is equal to the power calculated from the frequency domain representation of it. Hence, this means it is possible to compute a time interval around the pulse peak point, $\pm \Delta t$, that contains a defined (significant) proportion of the pulse energy, $\eta$, that is,

$$
\int_{t_{\text {peak }}-\Delta t}^{t_{\text {peak }}+\Delta t} p_{\text {cal }}{ }^{2}(t) d t=\frac{T \eta}{2} \sum_{n=1}^{2 u}\left(d_{n}\left|c_{n}\right|\right)^{2} .
$$

Finally, Table V contains the resulting characteristics of the proposed measuring receiver calibration pulse waveforms for CISPR bands $\mathrm{A}, \mathrm{B}$ and $\mathrm{C} / \mathrm{D}$.

Regarding the pulse area shown in Table $\mathrm{V}$, values were calculated using the trapezoidal integration rule within the time interval $\left[t_{\text {peak }}-\Delta t, t_{\text {peak }}+\Delta t\right]$ corresponding to the $99.9 \%$ of the pulse energy. The impulse area numerically obtained for the

Fig. 10. Calibration pulse for CISPR band B and its frequency spectrum. 
proposed pulse calibration waveforms are in conformity with the requirements for CISPR bands $\mathrm{A}, \mathrm{B}$, and $\mathrm{C} / \mathrm{D}$, respectively.

On the other hand, Table $\mathrm{V}$ also shows that the resulting pulse's spectrum flatness is according to the target set to the optimization problem, which was $1.5 \mathrm{~dB}$. Consequently, this means the synthesized pulses are compliant with the $\pm 2 \mathrm{~dB}$ requirement of pulse spectrum uniformity. Likewise, the numerical results of the impulse area calculation are coherent with the standard requirement. In this regard, the largest deviation of the impulse area was less than $-0.03 \mathrm{~dB}$ (for CISPR Band A) which is significantly better than the tolerance allowed by the standard $( \pm 0.5 \mathrm{~dB})$. Moreover, it is also verified the spectrum of the pulses decay $10 \mathrm{~dB}$ at twice the upper frequency of the corresponding band.

TABLE V. CHARACTERISTICS OF THE NUMARICALLY EVALUATED CALIBRATION PULSES

\begin{tabular}{r|c|c|c}
\hline \hline \multirow{2}{*}{ Parameter } & \multicolumn{3}{|c}{ CISPR Band } \\
\cline { 2 - 4 } & A & B & C/D \\
\hline \hline Max. pulse amplitude, $U_{\text {peak }}[\mathrm{V}]$ & 6.171 & 28.900 & 134.39 \\
\hline Peak-peak amplitude, $U_{p p}[\mathrm{~V}]$ & 6.823 & 31.956 & 148.71 \\
\hline Rise time ${ }_{10-90 \%} /$ Fall time $90-10 \%$ & $1.35 \mu \mathrm{s}$ & $6.75 \mathrm{~ns}$ & $0.20 \mathrm{~ns}$ \\
\hline Pulse duration, $2 \Delta t$, for $\eta=0.999$ & $54.53 \mu \mathrm{s}$ & $272.67 \mathrm{~ns}$ & $8.16 \mathrm{~ns}$ \\
\hline Impulse area, $A_{\text {imp }}[\mu \mathrm{Vs}]$ & 13.47 & 0.316 & 0.044 \\
\hline Pulse flatness, $F_{l, u}[\mathrm{~dB}]$ & 1.5007 & 1.5115 & 1.4935 \\
\hline
\end{tabular}

Another characteristic aspect of the proposed calibration pulses is their waveforms are equal when normalized in amplitude and in duration, as it is shown in Fig. 12.

Having a common waveform for the calibration pulses is important because this means the area of the normalized waveform is a constant value, explicitly, $A_{\text {norm }}=0.08$. Then, the calibration pulses for each band, and therefore the required pulse area, are scaled accordingly,

$$
A_{\text {imp }}=\left(U_{\text {peak }} \Delta t\right) A_{\text {norm }} .
$$

In fact, (15) means that, if the reference pulse waveform is generated with enough fidelity, the calibration of $A_{\text {imp }}$ can be achieved by a simple measurement of the peak voltage and the pulse duration.

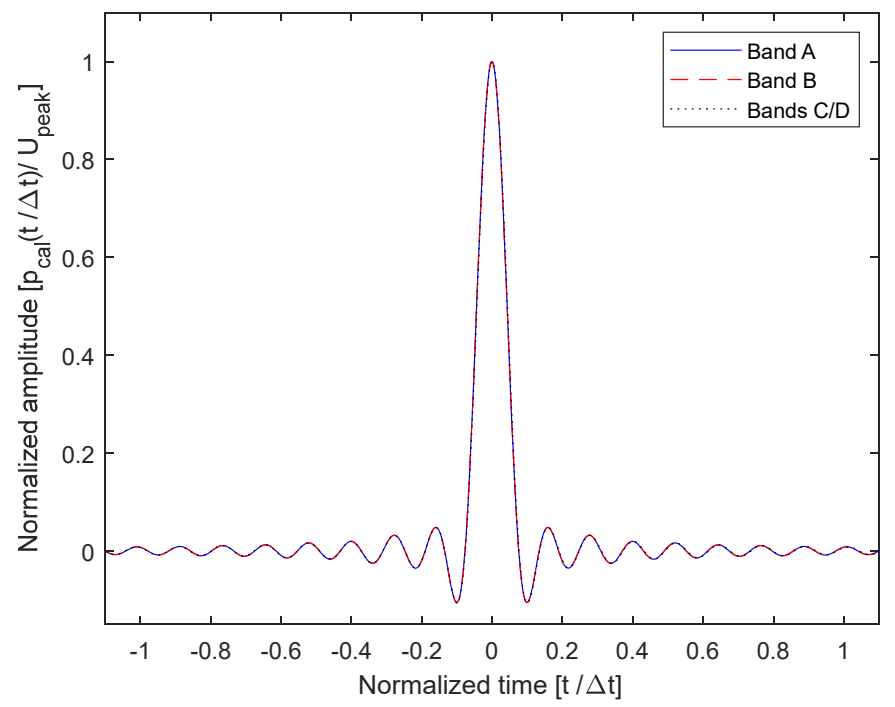

Fig. 12. Normalized calibration pulse waveform.

\section{EXPERIMENTAL VALIDATION}

For the experimental validation, the test setup in Fig. 13 was employed. On the one hand, the objective of the experiment was to verify if an arbitrary waveform generator can reproduce the synthetized calibration pulses accurately enough in terms of the pulse shape. On the other hand, the experiment was also intended to assess the response to pulses of a CISPR 16-1-1 compliant test receiver and corroborate the pulse spectrum flatness and band limited characteristics.

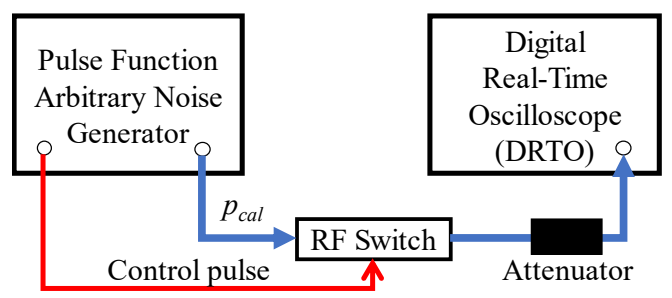

Fig. 13. Normalized calibration pulse waveform.

The proposed calibration pulse waveforms were generated with the 81150A Pulse Function Arbitrary Noise Generator (AWG) from Agilent, which has a bandwidth of $500 \mathrm{MHz}$, a resolution of the digital to analog converter (DAC) of 14 bits and waveform sample rate of $2 \mathrm{GSa} / \mathrm{s}$. One of the channels (CH1) of the AWG delivers the calibration pulses at intervals equal to the pulse duration. This is equivalent to repetition frequency higher than the required by the standard. Therefore, the reference pulse signal must be gated by using a control signal $(\mathrm{CH} 2)$ that turn on and off an RF switch placed between the AWG and the measuring receiver. Then, the pulse repetition frequency is set by the control signal. Regarding the measuring instrument, a real-time digital oscilloscope Tektronix DPO5104B with the input impedance set to $50 \Omega$ was used and the acquired waveforms were transformed into a quasi-peak amplitude level according to the methodology used compliant full time-domain EMI measurement systems [2], [3], [10], [12], [13]. Validation measurements were performed with an R\&S ESPI test receiver.

Due to limitations in the AWG, it was only possible to generate the calibration pulse for the bands A and B. Nonetheless, the validity of the concept and the experimental methodology could be applied for CISPR bands C/D provided an AWG of higher performance.

Regarding the waveform shape of the calibration pulse, Fig. 14 shows there is an excellent agreement between the numeric results and measurements. In terms of the normalized waveform, there is no noticeable difference in the shape between pulses obtained in bands A and B. An absolute mean error of $0.35 \%$ and $0.47 \%$ in the amplitude of the sampled waveform points was obtained for bands $\mathrm{A}$ and $\mathrm{B}$, respectively. Concerning the pulse area, a deviation of $0.32 \mathrm{~dB}$ and $0.53 \mathrm{~dB}$ with respect the normalized value was encountered for bands A and B, respectively. Such deviations may be attributed to a minor offset in the generated waveforms.

The spectrum of the calibration pulses exhibits a compliant behavior with respect to their flatness, as it is shown in Figure 
15 and 16 . The distortion observed in the spectrum is caused by the combined effect of the limited resolution in the amplitudes of both, the AWG's DAC, and the ADC of the oscilloscope.

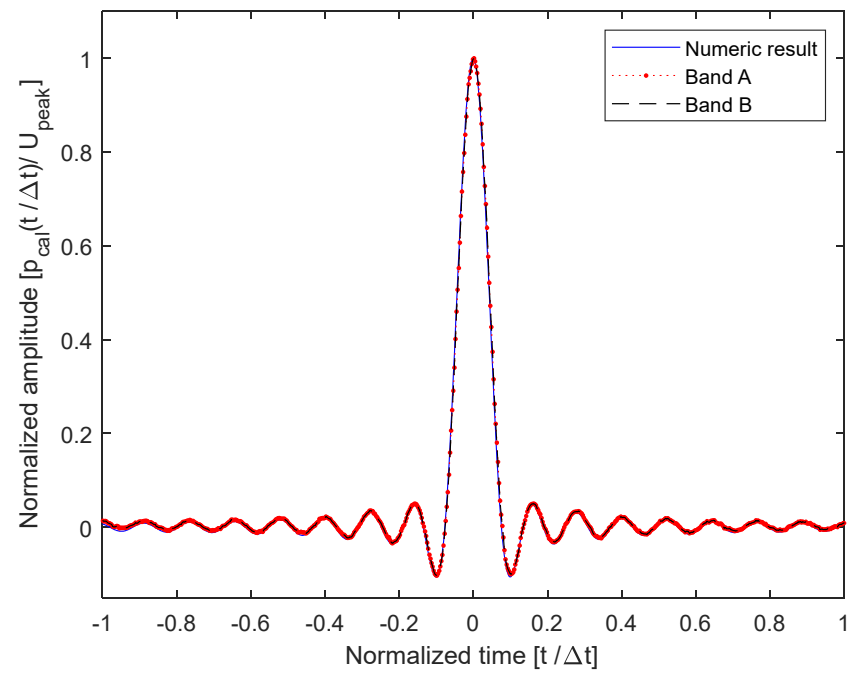

Fig. 14. Validation of the waveform shape of the calibration pulse.

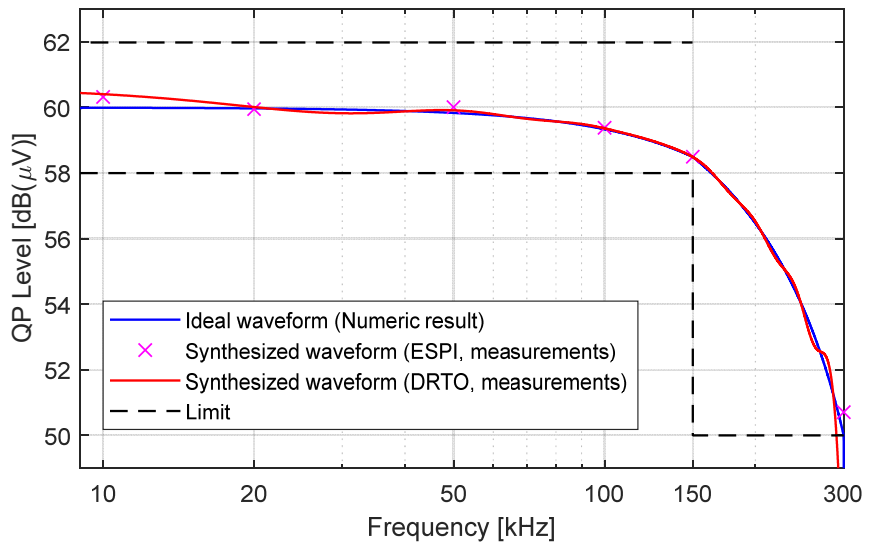

Fig. 15. Validation of the spectrum of the calibration pulse in band A.

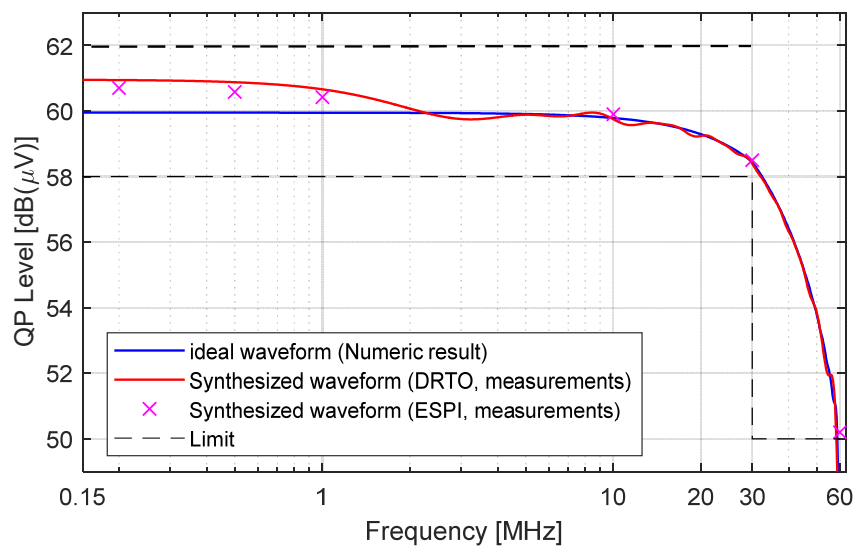

Fig. 16. Validation of the spectrum of the calibration pulse in band B.

A comparison between the quasi-peak level of the proposed pulses and the corresponding ones from the Schwarzbeck IGUU 2916 is presented in Table VI. The results are given only for those frequencies at which calibration data was available for the above-mentioned Calibration-Pulse Generator. In this regard, the flatness of both types of pulses is compliant with the standard requirement. Nonetheless, the spectrum decay outside the measurement band is not granted for the Schwarzbeck IGUU 2916, as indicated in Table II. However, calibration data for frequencies above the upper limit of the corresponding CISPR band is not available.

TABLE VI. QP LEVEL OF THE PROPOSED CALIBRATION PULSES VERSUS THE QP LEVEL OF THE SCHWARZBECK IGUU 2916 PULSES.

\begin{tabular}{|c|c|c|c|}
\hline \multirow{2}{*}{ Band } & \multirow{2}{*}{ Frequency } & \multicolumn{2}{|c|}{ QP level $[\mathbf{d B}(\boldsymbol{\mu} \mathbf{V})]$} \\
\cline { 2 - 4 } & & Proposed method & IGUU 2916 \\
\hline \multirow{4}{*}{$\mathrm{A}$} & $9 \mathrm{kHz}$ & 60.4 & 59.82 \\
\cline { 2 - 4 } & $10 \mathrm{kHz}$ & 60.4 & 59.75 \\
\cline { 2 - 4 } & $50 \mathrm{kHz}$ & 59.9 & 59.69 \\
\cline { 2 - 4 } & $100 \mathrm{kHz}$ & 59.4 & 59.71 \\
\cline { 2 - 4 } & $150 \mathrm{kHz}$ & 58.5 & 59.71 \\
\hline \multirow{4}{*}{$B$} & $150 \mathrm{kHz}$ & 60.9 & 59.59 \\
\cline { 2 - 4 } & $600 \mathrm{kHz}$ & 60.8 & 59.38 \\
\cline { 2 - 4 } & $1 \mathrm{MHz}$ & 60.6 & 59.41 \\
\cline { 2 - 4 } & $10 \mathrm{MHz}$ & 59.9 & 59.42 \\
\cline { 2 - 4 } & $30 \mathrm{MHz}$ & 58.5 & 59.23 \\
\hline
\end{tabular}

\section{CONCLUSIONS}

Departing from the scarce specifications given in the standard CISPR 16-1-1 regarding the characteristics of the reference pulses used for calibrating the measuring receiver response to pulses, a parametric model was developed and solved numerically to provide the complete waveform characteristics required to describe a compliant set of calibration pulses corresponding to CISPR bands A to D.

On the one hand, the approach followed in the paper can be used for generating suitable calibration pulses using arbitrary waveform generators as an alternative to the dedicated pulse generators for EMI test receiver calibration. On the other hand, it allows for a more complete theoretical definition of certain types of waveforms that are appropriate for calibration of the response to pulses of the measuring receivers.

In that sense, the presented results do not prevent or exclude other equally valid calibration pulses that may also fulfill the requirements of CISPR 16-1-1. Yet, our method and results could be used for comparison purposes. In this regard, when characterizing the output waveform of a calibration pulse generator, it would be beneficial to have a prior knowledge of the expected waveform to be measured.

Finally, the results could be employed to determine the specifications of sampling oscilloscope intended to characterize the waveforms of EMI calibration pulses with enough fidelity.

\section{REFERENCES}

[1] IEC CISPR, 16-1-1 ed4.0: Specification for radio disturbance and immunity measuring apparatus and methods - Part 1-1: Radio disturbance and immunity measuring apparatus - Measuring apparatus. IEC, 2015.

[2] M. A. Azpúrua et al., "Waveform Approach for Assessing Conformity of CISPR 16-1-1 Measuring Receivers," IEEE Trans. Instrum. Meas., vol. 67, no. 5, pp. 1187-1198, 2018.

[3] M. A. Azpúrua, M. Pous, J. A. Oliva, and F. Silva, "Fast and automated verification of multi-channel full time-domain EMI measurement system," in 2017 IEEE International Instrumentation and Measurement Technology Conference (I2MTC), 2017, pp. 1-6.

[4] CISPR/CIS/A - Radio-interference measurements and statistical methods, Interpretation sheet 1 - Specification for radio disturbance and immunity measuring apparatus and methods - Part 1-1: Radio disturbance and 
immunity measuring apparatus - Measuring apparatus. 2018.

[5] I. Agilent Technologies, Calibration Guide. 8590L Spectrum Analyzer. USA, 2001

[6] Fluke, "A Guide to Calibrating Your Spectrum Analyzer," 2006.

[7] I. Agilent Technologies, Calibration Guide. Agilent Technologies EMC Analyzers. USA, 2012.

[8] R. D. Drosd, "Transmission line pulse generator," 1956.

[9] C. R. Paul, Introduction to Electromagnetic Compatibility: Second Edition. 2006.

[10] M. A. Azpúrua, M. Pous, S. Çakir, M. Çetinta, and F. Silva, "Improving time-domain EMI measurements through digital signal processing," IEEE Electromagn. Compat. Mag., vol. 4, no. 2, pp. 82-91, 2015.

[11] M. A. Azpúrua, M. Pous, and F. Silva, "A measurement system for radiated transient electromagnetic interference based on general purpose instruments," in IEEE International Symposium on Electromagnetic Compatibility (EMC), 2015, vol. 2015-Septm.

[12] M. Pous et al., "Benefits of full time-domain EMI measurements for large fixed installation," in IEEE International Symposium on Electromagnetic Compatibility, 2016, vol. 2016-Novem, pp. 514-519.

[13] I. Setiawan, C. Keyer, M. Azpurua, F. Silva, and F. Leferink, "Timedomain measurement technique to analyze cyclic short-time interference in power supply networks," in 2016 Asia-Pacific International Symposium on Electromagnetic Compatibility (APEMC), 2016, vol. 01, pp. 279-282.

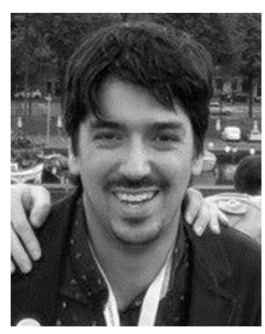

Marco A. Azpúrua (S'07-M'13-SM'-19) received the B.Sc. degree in telecommunications engineering, in 2008, and the M.Sc. degree in electrical engineering from the Universidad Central de Venezuela, Caracas, in 2013. In 2018, received the $\mathrm{Ph} . \mathrm{D}$. degree in Electronics Engineering from the Universitat Politècnica de Catalunya (UPC), Barcelona, Spain, for its contributions to Full Time Domain EMI measurements and their applications in complex scenarios. Currently, he is working as Researcher with the Electromagnetic Compatibility Group (GCEM) of the UPC. Formerly, he was a Researcher in the Applied Electromagnetics Laboratory, Instituto de Ingeniería (FII), Caracas-Venezuela. His research interests include electromagnetic compatibility, antenna and microwave measurement technologies, estimation of measurement uncertainty in complex systems and validation methods.

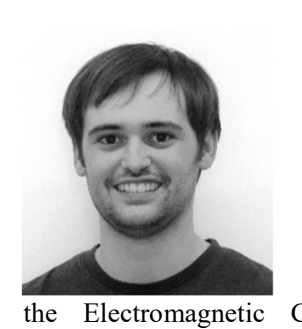

Marc Pous was born in Barcelona, Spain, in 1983. He received the M.Sc. degree in telecommunications engineering and the Ph.D. degree in radiated transient interferences and digital communication systems evaluation from the Universitat Politècnica de Catalunya, Barcelona, Spain, in 2009 and 2015, respectively. From 2003 to 2006, he was with the LGAI Technological Centre, Department of Electromagnetic Compatibility. In 2006, he was with the Electromagnetic Compatibility Group, Universitat Politècnica de Catalunya, where he has been participating in international and national research projects related to automotive, aerospace, railway, and medical industries. His research interests include the development of time domain measurement techniques to capture interferences which are not properly measured following the harmonized EMC standards.

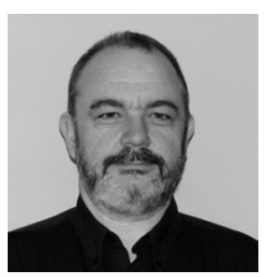

Ferran Silva (M'76-SM'81-F'87) received the M.Sc. and Ph.D. degrees from the Universitat Politècnica de Catalunya (UPC), Barcelona, Spain, in 1989 and 1997, respectively. Since 2000, he has been the Director of the Electromagnetic Compatibility Group, Universitat Politècnica de Catalunya (GCEMUPC) performing technology transfer activities to the industrial sector. $\mathrm{He}$ is currently an Associate Professor of Electronics with the Department of Electronic Engineering, UPC. His research interests include electromagnetic compatibility (EMC) in near field and time domain, including transients, with application to automotive, medical systems and installations. He has made more than 90 publications in journals and conferences and contributed to the Wiley Encyclopedia of Biomedical Engineering. He has participated in 14 research projects related to EMC. Dr. Silva is a Member of the IEEE EMC Society and the former Head of the Spanish chapter of the same society; he is also Member of the Spanish Standardization Committees SCTC77-210 and the CTN208 SCCISPR210A. He was/is the Chairman of the EMC Europe 2006/2019 International Symposium. Since then, he belongs to the Board of Chairmen of EMC Europe. 